\title{
Imaging with milli-XRF Benchtop Instrumentation
}

\author{
B.E. Scruggs*, R.W. Anderhalt*, L.I. Herczeg*, A. Lee* \\ * EDAX, 91 McKee Drive, Mahwah, NJ 07430
}

Imaging with milli-EDXRF (a.k.a. micro-XRF) benchtop instrumentation has been done for approximately 20 years with some recent examples showing the power of reflective X-ray optics [1, $2,3]$. This type of instrumentation uses a micro-focus X-ray tube and some form of X-ray optics, e.g. glass reflective optics or collimating optics, to analyze a restricted area of the sample $[4,5]$. Benchtop reflective X-ray optics are generally comprised of glass capillaries which allow for semiparallel beams or high intensity convergent beams. X-ray excited spectra contain additional information in the form of scattering peaks, i.e. Rayleigh and Compton, and reflections from crystalline phases, i.e. Bragg diffraction. In milli-XRF, the exciting beam can be filtered to increase $\mathrm{P}: \mathrm{B}$ or remove scattering and diffractive peak overlaps with elemental peaks. Typical filters are comprised of $\mathrm{Al}, \mathrm{Ti}, \mathrm{Ni}$ and $\mathrm{Zr}$ for example and range in thickness from 10 to $100 \mu \mathrm{m}$. The filter can be positioned before or after the X-ray optic. Positioning the filter between the X-ray tube and the X-ray optic maintains the focus of the X-ray beam on the sample. EDXRF has always been used for improved trace analysis with respect to SEM-EDS for elemental peaks in the range of 3 to $4 \mathrm{keV}$ and higher due to the lower Brehmsstrahlung and sensitivity can be improved further with filtering. Mapping with improved sensitivity for higher energy elemental line series can be achieved with milli-XRF. Figures 1 and 2 show an example of the use of filtered excitation in the elemental imaging of a meteorite cross-section to remove interference from Bragg diffractive peaks.

Analysis of phases within X-ray elemental maps generated with electron beam excitation [6] and Xray beam excitation [1] have been reported for quite some time. The phase analysis of X-ray excited elemental images can be more difficult given the additional peak forming phenomena which can occur. If the goal of the analysis is to report phases based on elemental composition, scattering and diffractive phenomena could overly complicate phase analysis results. Phase analysis can be applied to elemental images targeting only the labeled elemental peaks to provide useful, simplified results.

\section{References}

[1] T.C. Miller and G.J. Havrilla, Adv X-ray Anal 48 (2005) 274.

[2] J.M. Davis and D.E. Newbury, Microsc Microanal 15(Suppl. 2) (2008) 1084.

[3] J.M. Davis et al., Microsc Microanal 15(Suppl. 2), (2009) 542.

[4] J.A. Nicolosi et al., Adv X-ray Anal 41 (1999) 227.

[5] B.E. Scruggs et al., Adv X-ray Anal 42 (2000) 19.

[6] P.G. Kotula et al., Microsc Microanal 9 (2003) 1. 


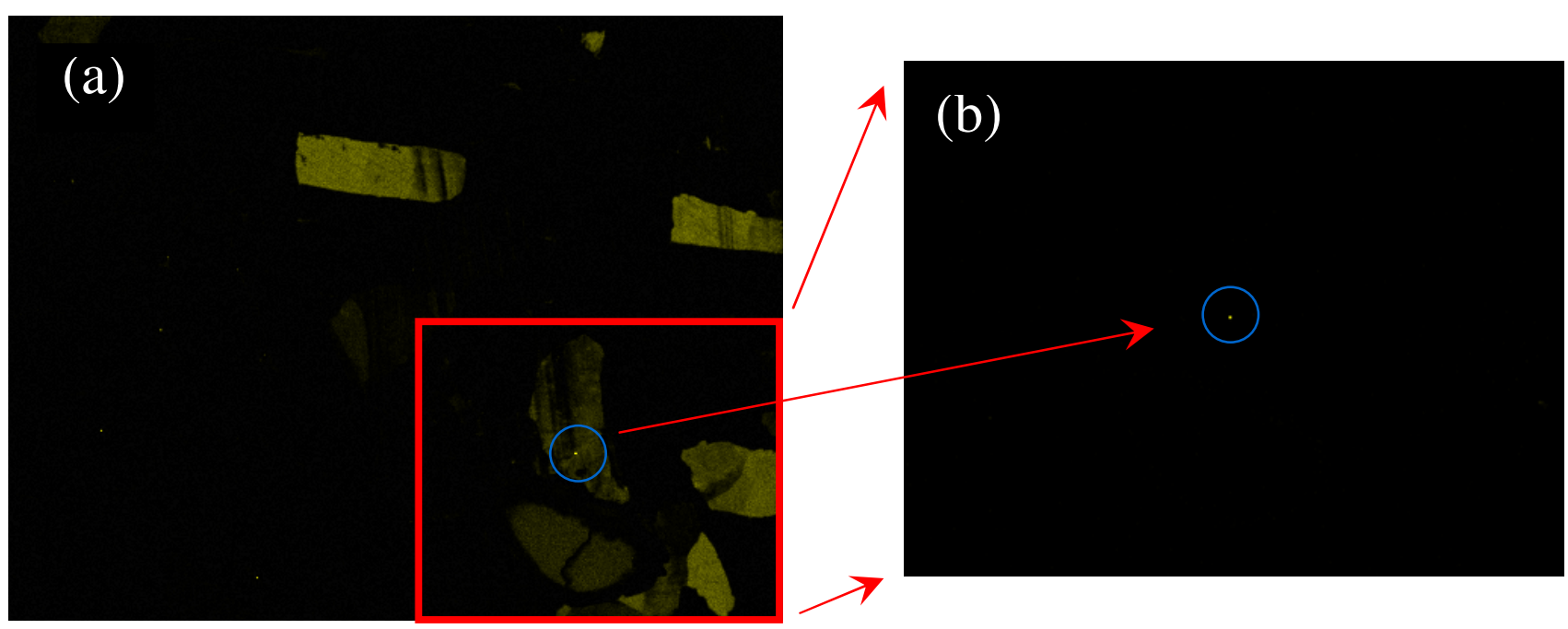

Figure 1a: Left hand image is a $\mathrm{Zn}(\mathrm{K})$ map $20 \mathrm{~mm}$ by $16 \mathrm{~mm}$ with a resolution of nominally $45 \mu \mathrm{m}$ of an Odessa meteorite cross-section using milli-XRF. Large crystal grains are observed due to Bragg diffraction reflections into the detector.

Figure 1b: In the right hand image, the exciting beam has been filtered with a Ni filter, removing the exciting energy in the range of $\mathrm{Zn}(\mathrm{K})$. The $\mathrm{Zn}(\mathrm{K})$ map of this section of the meteor is shown. Bragg diffractive reflections are eliminated allowing a more clear view of the $\mathrm{Zn}$ inclusion.

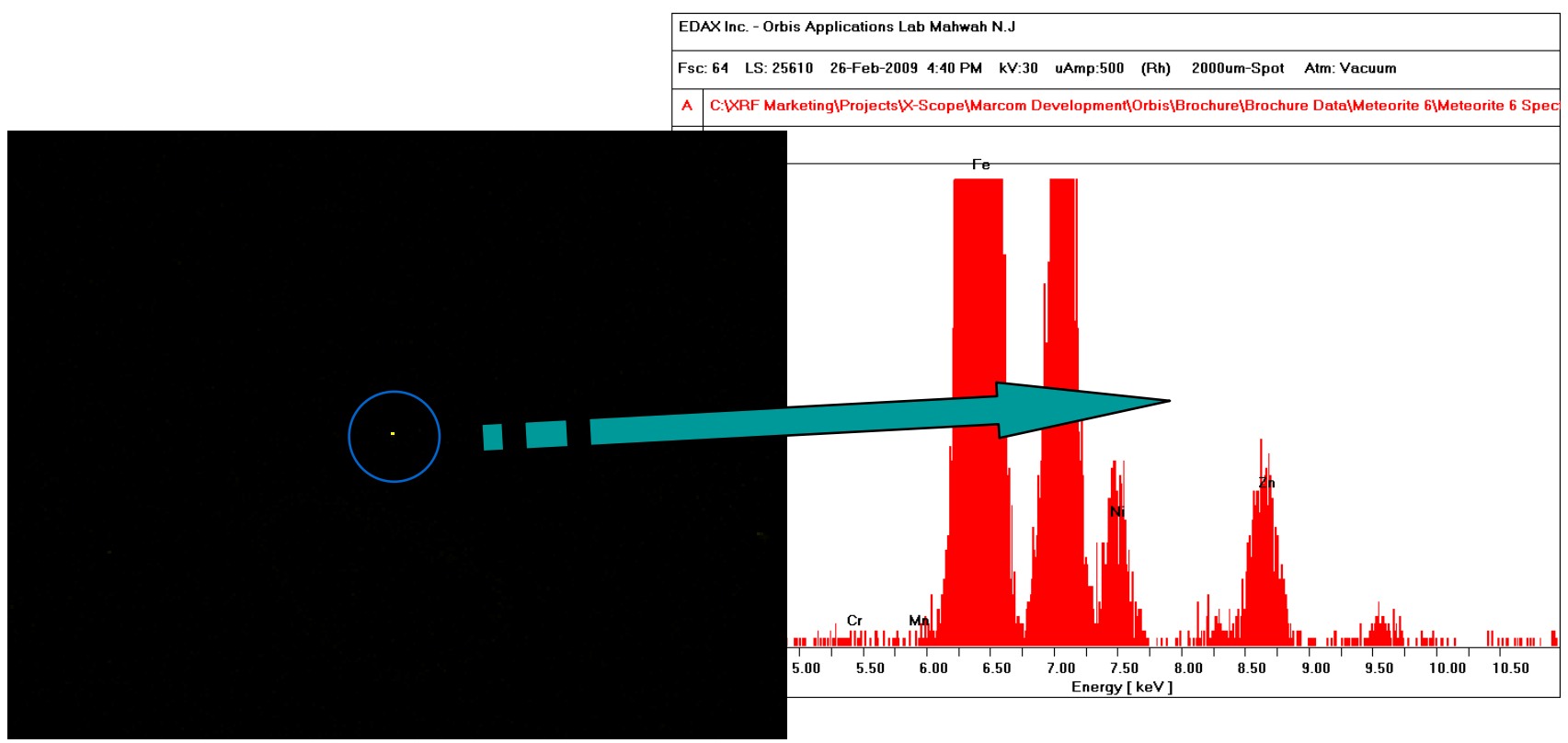

Figure 2: In the left hand portion of the figure, the $\mathrm{Zn}(\mathrm{K})$ map with Ni-filtered excitation shown in Figure 1a. Spectral review of the bright pixels in the right hand portion of the image confirms the $\mathrm{Zn}(\mathrm{K})$ peaks with both alpha and beta $\mathrm{K}$ lines observed. 